\title{
Dedicated to Professor Kazuhiko Mizuno on the occasion of his retirement
}

\author{
Gen-ichi Konishi • Hiroshi Ikeda
}

Published online: 15 June 2012

(C) Springer Science+Business Media B.V. 2012

This Special issue of Research on CHEMICAL INTERMEDIATES (RCI) are dedicated to Professor Kazuhiko Mizuno of Osaka Prefecture University, Japan, on the occasion of his retirement. During his long and productive career, Professor Mizuno conducted pioneering studies in synthetic organic photochemistry. His efforts led to the development of many novel photochemical processes that occur through a variety of reaction intermediates, including singlet or triplet excited states, excimers, exciplexes, radical cations, radical anions, radicals, and biradicals. The work has been reported in more than 250 publications.

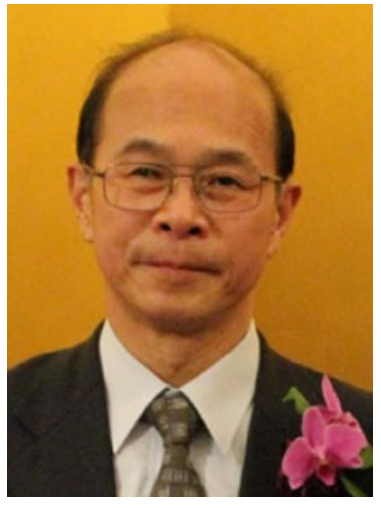

\section{G. Konishi}

Department of Organic and Polymeric Materials, Tokyo Institute of Technology,

O-okayama, Meguro-ku, Tokyo 152-8552, Japan

e-mail: konishi.g.aa@m.titech.ac.jp

H. Ikeda $(\bowtie)$

Department of Applied Chemistry, Graduate School of Engineering, Osaka Prefecture University, 1-1 Gakuencho, Naka-ku, Sakai, Osaka 599-8531, Japan

e-mail: ikeda@chem.osakafu-u.ac.jp 


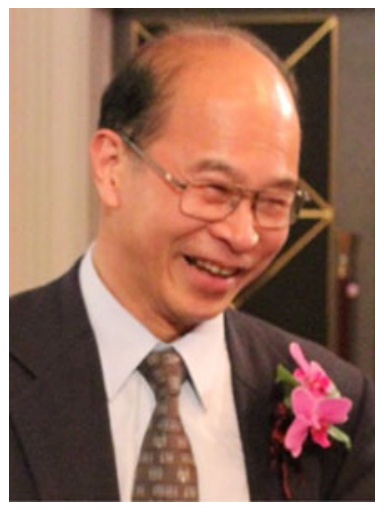

Professor Mizuno was born in Osaka, Japan in 1947 and graduated from the Department of Applied Chemistry of Osaka University with a BT in 1971 and a PhD in 1976 under the supervision of Professors S. Tsutsumi and H. Sakurai, respectively. He began his academic career at Osaka Prefecture University (OPU), Sakai, in the laboratories of Professors Y. Otsuji and H. Inoue, and remained at that institution until March 31, 2012 when he retired and became a Professor Emeritus. In the early stages of his career (1979-1980), Professor Mizuno performed studies as a postdoctoral fellow at the University of Texas at Dallas in Professor R. A. Caldwell's laboratory. He also spent three months at the University of California, Santa Barbara, with Professor R. J. Watts in 1987, as a US-Japan Exchange Researcher. Professor Mizuno received the prestigious Japanese Photochemistry Association Award in 1996, and the Incentive Award in Synthetic Organic Chemistry, Japan, in 1986. Professor Mizuno is now serving as a guest professor at the Nara Institute of Science and Technology (NAIST).

Professor Mizuno's research interests focused on synthetic aspects of organic photochemistry. Early in his career (1980s) he discovered that special salts (e.g., $\left.\mathrm{Mg}\left(\mathrm{ClO}_{4}\right)_{2}\right)$ enhance the efficiency of oxygenation reactions promoted by photoinduced electron transfer (PET) and that occur via radical cation intermediates. In addition, his efforts led to the development of a prototype of a novel, tandem, three-component coupling reaction, triggered by PET between allyltin compounds, electron-poor alkenes, and alkyl iodides. This process has become known as the "Mizuno-Otsuji three-component radical coupling reaction". During the past decade, work directed by Professor Mizuno led to the development of a highly concise, one-step synthetic method for preparation of triquinanes, substances that contain a complicated polycyclic framework found in interesting natural products. The key process used in this route involves stereo-specific and region-specific intramolecular $[3+2]$ photocycloaddition of naphthalene-ethene/ethyne dyads tethered by a three-atom chain. The high specificity of this reaction is a consequence of the formation of an intramolecular exciplex.

All who have interacted with Professor Mizuno know he is a gentle, friendly, and kind person, and that, in addition to chemistry, he loves sport, especially volleyball and baseball. Although a few years ago he suffered from a heart ailment, surgery 
cured this problem and left him literally "young at heart". As a result, he is eager to continue his research work at NAIST.

It would be remiss not to mention that Professor Mizuno's accomplishments in chemistry were made possible by the generous understanding and devoted cooperation of his family, especially his lovely wife, Mari. Retirement from the Osaka Prefecture University will mean that he will now be able to spend more time with his family.

All of his colleagues congratulate Professor Mizuno on his outstanding career in chemistry and wish him and his family a long, productive, and enjoyable life.

Finally, we would like to acknowledge the RCI Editor-in-Chief, Professor Masakazu Anpo (OPU), and Springer Production Editor Balaji Sundarrajan for their assistance in preparing in these special issues, and the authors and reviewers who have made invaluable contributions. 